\title{
Equivalency of Sleep Estimates: Comparison of Three Research-Grade Accelerometers
}

\author{
Tatiana Plekhanova, Alex V. Rowlands, and Tom Yates \\ University of Leicester
}

\author{
Andrew Hall \\ University Hospitals of Leicester NHS Trust
}

\author{
Emer M. Brady, Melanie Davies, Kamlesh Khunti, and Charlotte L. Edwardson \\ University of Leicester
}

\begin{abstract}
Introduction: This study examined the equivalency of sleep estimates from Axivity, GENEActiv, and ActiGraph accelerometers worn on the nondominant and dominant wrists and with and without using a sleep log to guide the algorithm. Methods: 47 young adults wore an Axivity, GENEActiv, and ActiGraph accelerometer continuously on both wrists for 4-7 days. Sleep time, sleep window, sleep efficiency, sleep onset, and wake time were produced using the open-source software (GGIR). For each outcome, agreement between accelerometer brands, dominant and nondominant wrists, and with and without use of a sleep log, was examined using pairwise $95 \%$ equivalence tests ( $\pm 10 \%$ equivalence zone) and intraclass correlation coefficients (ICCs), with 95\% confidence intervals and limits of agreement. Results: All sleep outcomes were within a 10\% equivalence zone irrespective of brand, wrist, or use of a sleep log. ICCs were poor to good for sleep time (ICCs $\geq .66$ ) and sleep window (ICCs $\geq .56$ ). Most ICCs were good to excellent for sleep efficiency (ICCs $\geq .73$ ), sleep onset (ICCs $\geq .88$ ), and wake time (ICCs $\geq .87$ ). There were low levels of mean bias; however, there were wide $95 \%$ limits of agreement for sleep time, sleep window, sleep onset, and wake time outcomes. Sleep time (up to $25 \mathrm{~min}$ ) and sleep window (up to $29 \mathrm{~min}$ ) outcomes were higher when use of the sleep log was not used. Conclusion: The present findings suggest that sleep outcomes from the Axivity, GENEActiv, and ActiGraph, when analyzed identically, are comparable across studies with different accelerometer brands and wear protocols at a group level. However, caution is advised when comparing studies that differ on sleep log availability.
\end{abstract}

Keywords: ActiGraph, Axivity, free-living, GENEActiv, raw acceleration, sleep duration

A large body of evidence has emerged implicating short sleep duration, poor sleep quality, and later timing of sleep (associated with being an evening chronotype) in all-cause mortality (Knutson \& von Schantz, 2018) and in cardiovascular and cardiometabolic risk factors (Reutrakul \& Van Cauter, 2018). The majority of studies linking poor sleep with negative health outcomes often rely on a single, retrospective self-report of sleep, which is unreliable and sensitive to recall bias (Lauderdale, Knutson, Yan, Liu, \& Rathouz, 2008). Moreover, self-reported sleep has been demonstrated to depend on personal and sleep characteristics such as weight status, ethnicity, and habitual sleep duration of participants (Lauderdale et al., 2008). Polysomnography (PSG) is considered the gold standard for sleep assessment. Nevertheless, PSG is impractical, expensive, and labor-intensive and hence not feasible for large-scale studies (Zinkhan et al., 2014). In sleep research, actigraphy - a device worn on the wrist, which resembles a watchhas been used widely and validated against PSG in both community and clinical samples (Sadeh, 2011). Wrist actigraphy allows sleep assessment over several days and measures daily sleep-wake cycles (Berger et al., 2008; Martin \& Hakim, 2011). However, the data are in the form of manufacturer-specific activity "counts"

Plekhanova is with the Diabetes Research Centre, Leicester General Hospital, University of Leicester, Leicester, United Kingdom. Rowlands, Yates, Brady, Davies, Khunti, and Edwardson are with the University of Leicester, Leicester, United Kingdom. Hall is with the University Hospitals of Leicester NHS Trust, Leicester, United Kingdom. Plekhanova (tp177@le.ac.uk) is corresponding author. over a specific time window, making it difficult to compare the data with different accelerometer brands (van Hees et al., 2015). Therefore, there is a need for an objective and simple method of sleep assessment to facilitate the comparison and pooling of data across studies.

Recently, wrist-worn accelerometers have become increasingly used for objective measurement of physical activity in large population-based studies, such as UK Biobank (Doherty et al., 2017), the U.S. National Health and Nutrition Examination Survey (Loprinzi \& Cardinal, 2011), and the British Whitehall II study (Menai et al., 2017), where they were worn $24 \mathrm{hr} /$ day to maximize compliance (Zinkhan et al., 2014). This enables the objective measurement of physical behaviors (physical activity, sedentary time, and sleep) across day and night, presenting wrist-worn accelerometers as a potential tool for sleep research as well as physical activity research (Rosenberger, Buman, Haskell, McConnell, \& Carstensen, 2016). The latest generation of accelerometers measure acceleration at high resolution in universal units of gravitational acceleration (raw accelerometry), potentially improving comparability among different brands of accelerometers and allowing more control in the data processing (van Hees et al., 2015). There are a number of accelerometer brands available; however, the three most widely used research-grade raw data accelerometer brands deployed in epidemiological studies are the Axivity (Axivity Ltd., Newcastle, United Kingdom), ActiGraph (ActiGraph LLC, Pensacola, FL), and GENEActiv (ActivInsights Ltd., Cambridgeshire, United Kingdom).

Various accelerometer proprietary sleep-wake scoring algorithms have been developed in response to differences in wear 
location and how acceleration data are processed (Borazio, Berlin, Kücükyildiz, Scholl, \& Van Laerhoven, 2014; Cole, Kripke, Gruen, Mullaney, \& Gillin, 1992; Sadeh, 2011). Choice of algorithm influences sleep-wake time estimates suggesting that sleep variables derived from different algorithms might not be comparable (Quante et al., 2018). van Hees et al. (2015) developed an algorithm based on angular wrist rotation. In this method, the arm angle is determined relative to the horizontal plane of the human body when the accelerometer is worn on the wrist, and low frequency of changes in the arm angle are used to indicate sleep. Arm angle is derived from the $z$ axis (the axis positioned perpendicular when the wrist is in the anatomical position), which is estimated from the median values of the three orthogonally positioned raw acceleration sensors in gravitational $(g)$ units $(1 g=$ $1,000 \mathrm{mg}$ ) derived based on a rolling 5-s time window. Estimated arm angle is then averaged per 5-s epoch and used to assess change in arm angle between successive 5-s epochs (van Hees et al., 2015). Sleep is defined as sustained inactivity detected as the absence of change in wrist rotation greater than $5^{\circ}$ for $5 \mathrm{~min}$, or user-defined duration, when participants self-report being asleep. This method showed $83 \%$ accuracy between sleep duration derived from an accelerometer and PSG in sleep clinic patients (van Hees et al., 2015). As with actigraphy, though, this approach requires the completion of a sleep log where participants record their sleep onset and waking times - data which are used to guide the algorithm to detect nocturnal sustained bouts of inactivity (Martin \& Hakim, 2011; van Hees et al., 2015). In addition to inaccurate self-report of sleep onset and wake time, the use of sleep logs in large-scale studies is time consuming for researchers and adds to the burden on participants (Girschik, Fritschi, Heyworth, \& Waters, 2012). Therefore, it is desirable to be able to classify sleep without the aid of sleep log data, to facilitate use in studies where a sleep log was not used, for instance, in the UK Biobank data set (Doherty et al., 2017). In this data set, over 100,000 participants wore Axivity AX3 wrist-worn triaxial accelerometers on their dominant wrists for 7 days (Doherty et al., 2017). Further work by van Hees et al. (2018) has led to the development of a sleep detection algorithm (the Heuristic Algorithm looking at Change of Z-Angle, HDCZA) to identify the sleep period time window (SPT window, which refers to the time window starting at sleep onset and ending when the person wakes up after the last sleep episode of the night) in the absence of sleep diaries from comparison with sleep diary records and relative to PSG. The SPT window was compared against the sleep diary in 3,752 participants (mean $\pm S D$ : 69.1 \pm 5.6) and was 10.9 and 2.9 min longer compared with the sleep diary in men and women, respectively (van Hees et al., 2018). Next, the SPT window from accelerometer data from 22 healthy sleepers (mean $\pm S D: 22.8 \pm 4.5$ years) and 28 sleep clinic patients (mean \pm $S D: 45.0 \pm 1.5$ years) was tested against PSG. Mean C-statistic and accuracy to detect the SPT window compared with PSG was 0.86 and $87 \%$ and 0.83 and $89 \%$ in clinic-based and healthy sleepers, respectively. Sensitivity to detect sleep within the SPT window was $92 \%$ and $93 \%$, in clinic-based and healthy sleepers, respectively (van Hees et al., 2018). However, it is worth noting that the algorithm was developed based on sleep data from a small sample of a mostly sleep clinic population, where, in seven patients, the algorithm deviated by longer than $2 \mathrm{hr}$. Additionally, the comparison of accelerometer sleep data with sleep diary records was derived from older adults, limiting the generalizability of the findings. While further validation of the HDCZA is warranted, this algorithm can be applied to raw accelerometer data irrespective of accelerometer brand and is available as part of the GGIR package in $\mathrm{R}$ [https://cran.r-project.org] (van Hees et al., 2014, 2019), which is open source and an efficient method for processing and analyzing raw data that can combine sleep and physical activity data. The algorithm can also be applied to data in the absence of sleep diary records.

With the growing availability of accelerometry data from three accelerometer brands for epidemiological research, often, with no sleep $\log$, it is necessary to establish whether sleep outcomes are comparable between the brands and with and without the use of sleep log data to guide the algorithm. If outcomes are comparable, it would be possible to pool or compare sleep data across these largescales studies deploying 24-hr accelerometry. Free-living measures of average dynamic acceleration, and physical activity outputs that depend on acceleration magnitude, tend to be lower at the nondominant relative to the dominant wrist and for the ActiGraph compared with the GENEActiv and the Axivity (Rowlands et al., 2019). However, outputs that take into account the distribution of data are more consistent across wrist and monitor brand (Rowlands et al., 2019). It is not known whether different brands of accelerometers can be considered equivalent for sleep outcomes or whether sleep outcomes can be considered equivalent between the nondominant wrist and the dominant wrist. This is important as, for instance, participants wore the Axivity on their dominant wrists in the UK Biobank study, whereas participants wore the GENEActiv and ActiGraph on their nondominant wrists in the British Whitehall II and National Health and Nutrition Examination Survey studies, respectively.

The aims of this study were to investigate the extent to which accelerometer sleep outcomes generated using the GGIR package are comparable:

(a) Between the same brand of accelerometer worn on the nondominant wrist and the dominant wrist

(b) Between different brands of accelerometers worn on the same wrist

(c) Between different brands of accelerometers worn on different wrists

(d) When using and not using a sleep log to guide the algorithm

\section{Methods}

A sample of 56 participants was recruited through the University of Leicester and Loughborough University between November 2017 and July 2018. The study was approved by the ethics representatives in the College of Life Science, University of Leicester. Prior to participation, all participants signed a written informed consent. Adults aged 18-85 years were invited to take part in the study. The main inclusion criteria were: ability to read written English and communicate in English. There were no exclusion criteria; information on any limits to physical mobility was recorded.

Participants' height and weight were assessed to the nearest $0.5 \mathrm{~cm}$ and $0.5 \mathrm{~kg}$, respectively. Handedness was self-reported by the participants, and each participant was fitted with a GENEActiv (ActivInsights Ltd.), Axivity AX3 (Axivity Ltd., Newcastle, United Kingdom), and ActiGraph GT9X (ActiGraph LLC, Pensacola, FL) on their right and left wrists and was asked to wear them $24 \mathrm{hr} /$ day for 7 days. It is recommended that at least 5 days are recorded for a sleep assessment (Sadeh, 2011). However, in this study, the objective was to establish the comparability of accelerometer sleep outcomes between outputs; thus, due to discomfort from the straps reported by the participants at the beginning of the study, the wear protocol was reduced to 4 days to ensure 
compliance. This resulted in high compliance, with an average of 4.5 days out of 4-7 days. To reduce the number of wrist straps worn for comfort reasons, the Axivity was taped to the ActiGraph, and the GENEActiv was placed to the side of the two monitors. The side was randomized between the participants but consistent between wrists for each participant. The setup of the monitors is shown in Supplementary Document S1 (available online). Positioning the GENEActiv adjacent to the ActiGraph, and when processed identically using GGIR, previously showed high agreement between brands for sleep outcomes, such as time in bed and sleep duration, with ICCs of .91-.99 and mean bias of $\pm 0.10 \mathrm{hr}$ for time in bed and ICCs of .97-.99 and mean bias of $\pm 0.01 \mathrm{hr}$ for sleep duration (Rowlands, Yates, Davies, Khunti, \& Edwardson, 2016). Additionally, physical activity outcomes from GGIR outputs were equivalent when worn on the nondominant wrist between monitor brands with Axivity taped to either GENEActiv (Rowlands et al., 2019) or ActiGraph (Rowlands et al., 2019). Alongside this, the participants completed a sleep log for each night, recording the time they woke up and fell asleep for the days they wore devices. If participants needed to remove the monitors for any reason, they were instructed to remove all the devices simultaneously and leave them on a stationary surface while they were not worn.

\section{Accelerometers}

The GENEActiv Original, Axivity AX3, and ActiGraph GT9X Link are triaxial accelerometers with a dynamic range of $\pm 8 g$, where $g$ is equal to the Earth's gravity. All accelerometers were configured to record at a frequency of $100 \mathrm{~Hz}$ and initialized using the same PC to match the time of the recording period. GENEActiv monitors were initialized, and the data were downloaded and saved in raw format as BIN files using GENEActiv PC software (version 3.2). Axivity devices were set up, and the data were downloaded with OmGui open-source software (OmGui, version 1.0.0.30; Open Movement, Newcastle, United Kingdom). ActiGraph Link GT9X monitors were initialized and downloaded using ActiLife (version 6.13.3), saved in raw format as GT3X, then converted to CSV format for data processing. Raw accelerometer data from the GT9X Link and the older version of ActiGraph-GT3X+ (commonly used in other studies) are reported to be highly correlated with correlations for mean acceleration in different intensities ranging between .71 and .96 (Montoye et al., 2018).

\section{Data Processing and Outcome Measures}

All accelerometer files were processed with and without a sleep log using GGIR package in $\mathrm{R}$ (version 1.5-21; https://cran.r-project. org/web/packages/GGIR/; van Hees et al., 2013, 2014). Importantly, raw data from the GENEActiv, Axivity, and ActiGraph can be processed identically in GGIR to produce sleep outcomes both using a sleep log and using the HDCZA (sleep detection algorithm) (van Hees et al., 2018). Signal processing in GGIR includes autocalibration using local gravity as a reference, detection of sustained abnormally high values, detection of nonwear, and calculation of the average magnitude of dynamic acceleration (i.e., the vector magnitude of acceleration corrected for gravity [Euclidean Norm minus $1 g]$ ) in milligravitational units averaged over 5-s epochs. A detailed description on how the algorithm works has been published in the aforementioned work by van Hees et al. (2018) (https://doi.org/10 .1038/s41598-018-31266-z). The GGIR script used can be found in Supplementary Document S2 (available online).

The following sleep measures were assessed: sleep time (accumulated nocturnal sustained inactivity bouts), sleep window (time difference between falling asleep and waking up, including time being awake during night), sleep onset, and wake time and sleep efficiency (ratio of sleep time compared with sleep window). Where sleep log was not used, the SPT window was derived from sleep onset and wake detected by the HDCZA. Where sleep log was used to guide the algorithm to detect the SPT window, sleep onset time was equal to the start of the first and wake time was equal to the end of the last sustained inactivity bout that overlapped or followed the sleep onset/wake time derived from the sleep log (van Hees et al., 2018). Sleep onset and wake time were analyzed as decimals, that is, $13: 15$ as 13.25 , and then converted into hours and minutes.

\section{Statistical Analyses}

Only those who provided a full set of data (all six valid files per participant: three monitors on both wrists) for at least one night (daily detected wear of at least 16 hr; da Silva et al., 2014; van Hees et al., 2015) were included in analyses. All variables were tested for normality by exploring the differences in distributions graphically using histograms and statistically using the Kolmogorov-Smirnov test. Descriptive statistics (mean $\pm S D$ ) or median (25th-75th percentile) where data were not normally distributed were calculated for all outputs. Pairwise $95 \%$ equivalence tests were used to determine whether the $95 \%$ confidence intervals (CI) for the mean of one accelerometer fell within a $10 \%$ equivalence zone of the second accelerometer, as used in previous studies comparing activity monitors (Boddy et al., 2018; Lee, Kim, \& Welk, 2014; Rosenberger et al., 2016; Rowlands et al., 2018). However, the $10 \%$ equivalence zone might be not strict enough when the values are very high; for example, a typical sleep duration might be $480 \mathrm{~min}$ with a $10 \%$ equivalence zone equating to $\pm 48 \mathrm{~min}$. Therefore, a stricter $5 \%$ equivalence zone was also used to evaluate the equivalency of all sleep outcomes between all combinations. Log transformation was applied to data that were not normally distributed. Log transforming the data allows hypotheses about ratios, that is, the percentage above or below the reference mean, to be analyzed; for example, a ratio of 1.0 indicates that the means are equal; a ratio of $0.9-1.1$ indicates that the mean is within $90 \%$ and $110 \%$ of the reference mean. Because no accelerometer is a gold standard for sleep measurement, equivalency tests were conducted with each accelerometer as a reference monitor in each accelerometer pairing. Sleep outcomes from accelerometer pairings were considered equivalent if equivalency was achieved with each accelerometer as a reference monitor. Equivalence results are shown with the Axivity as the reference accelerometer in the Axivity/GENEActiv and Axivity/ActiGraph pairings and the GENEActiv as the reference monitor in the GENEActiv/ActiGraph pairing. The level of agreement between outcome variables was examined using intraclass correlation coefficients (ICC, single measures, absolute agreement), with 95\% CI and limits of agreement (LoA; Bland \& Altman, 1986). The level of reliability from ICCs was based on the lower bound of the $95 \%$ CI of the ICC values. If lower bound of the $95 \%$ CI was $>.5$, reliability was classified as "poor," .5-.75 as "moderate," >.75-.9 as "good," and $>.9$ as "excellent" (Koo \& Li, 2016). Bland-Altman plots were created to assess the pattern and magnitude of differences in sleep estimates between each pairing of accelerometers. Descriptive statistics, ICCs, and LoA were performed using SPSS Statistics (version 24.0; IBM Corp., Armonk, NY), and equivalency tests were conducted in Minitab (version 17; Minitab, Inc., State College, PA). Alpha was set at .05. 


\section{Results}

A total of 56 participants took part in the study. Two participants did not wear their monitors during the night, and seven monitors malfunctioned, thus data from 47 participants (16 males, 31 females, seven left handed, age [mean $\pm S D$ ] $24.5 \pm 4.5$ years, body mass index $23.6 \pm 3.8 \mathrm{~kg} / \mathrm{m}^{2}$ ) were included in the analyses (Supplementary Table S1 [available online]). The minimum valid data provided by participants were 1 day and night and a maximum of 7 days and 6 nights.

Descriptive statistics of sleep outcomes (mean or median [25th-75th percentile per night]) by monitor are presented in Table 1.

\section{Between Wrists and Within Brand}

The sleep outcome results between the same brand of accelerometer worn on the nondominant wrist and the dominant wrist are shown in Table 2 and the Bland-Altman plots in Supplementary Figure S1 (available online). The mean differences between pairs of monitors for all sleep outcomes were low; however, 95\% LoA for sleep time, sleep window, sleep onset, and wake time were high. Reliability between nondominant and dominant wrists across all monitors for sleep onset and wake time was good to excellent, with ICC values of .88-.97. ICCs for all sleep outcomes for the Axivity were lower and LoA higher for sleep time (up to $\pm 86.7 \mathrm{~min}$ ), sleep window $( \pm 102.9 \mathrm{~min})$, sleep onset $( \pm 90.0 \mathrm{~min})$, and wake time $( \pm 78.0 \mathrm{~min})$ compared with the ActiGraph and GENEActiv. However, mean bias for the Axivity for sleep time $( \pm 3.5 \mathrm{~min})$, sleep window $( \pm 1.3 \mathrm{~min})$, sleep onset $( \pm 3.0 \mathrm{~min})$, and waking time $( \pm 1.2 \mathrm{~min})$ was low. The level of agreement between nondominant ActiGraph/dominant ActiGraph appeared higher compared with the other two monitor pairings. All sleep outcomes were within the $5 \%$ and $10 \%$ equivalence zones between pairs of monitors and are shown in Figure 1.

\section{Within Wrist and Between Brands}

The sleep outcome results between different brands of accelerometers when worn on the nondominant and dominant wrists are presented in Table 3, and Bland-Altman plots are presented in Supplementary Figure S2a and S2b (available online).

Nondominant placement. Reliability was good to excellent between all three pairs of accelerometers for all sleep outcomes when worn on the nondominant wrist, with ICCs of .85-.98.

Table 1 Descriptive Statistics of Sleep Outcomes Without/With Sleep Log (Mean, SD, or Median 25th-75th Percentile Were Not Normally Distributed per Night) by Monitor $(N=47)$

\begin{tabular}{|c|c|c|c|c|c|c|}
\hline \multirow[b]{2}{*}{ Sleep outcomes } & \multicolumn{3}{|c|}{ Nondominant wrist } & \multicolumn{3}{|c|}{ Dominant wrist } \\
\hline & Axivity & GENEActiv & ActiGraph & Axivity & GENEActiv & ActiGraph \\
\hline \multicolumn{7}{|l|}{ Without sleep log } \\
\hline Sleep time (min) & $411.8(49.2)$ & $416.8(49.7)$ & $416.6(48.9)$ & $415.3(60.8)$ & $422.5(50.4)$ & $414.9(47.2)$ \\
\hline Sleep window $(\mathrm{min})$ & $473.2(52.6)$ & $480.7(54.3)$ & $478.5(50.9)$ & $474.5(58.0)$ & $483.7(50.7)$ & $476.2(49.8)$ \\
\hline Sleep efficiency $(\%)$ & $87.1(4.1)$ & $86.9(4.5)$ & $86.8(4.2)$ & $88.3(84.3-91.3)$ & $87.5(4.7)$ & $87.6(4.6)$ \\
\hline Sleep onset (hr:min) & 00:12 (90) & 00:10 (90) & 00:05 (90) & 00:00 (23:00-01:05) & 00:05 (90) & 00:30 (78) \\
\hline Wake (hr:min) & 08:12 (90) & 08:20 (84) & $08: 20(84)$ & 08:15 (90) & 08:20 (84) & $08: 15(84)$ \\
\hline \multicolumn{7}{|l|}{ With sleep log } \\
\hline Sleep time (min) & $391.9(52.1)$ & $393.9(49.9)$ & $394.9(52.0)$ & $401.1(62.0)$ & $397.7(53.7)$ & $390.1(50.3)$ \\
\hline Sleep window $(\mathrm{min})$ & $450.7(56.0)$ & $453.0(55.3)$ & $450.8(54.0)$ & $458.5(58.4)$ & $454.9(57.1)$ & $451.8(51.3)$ \\
\hline Sleep efficiency $(\%)$ & $87.1(4.4)$ & $87.3(5.0)$ & $86.7(4.9)$ & $88.2(84.0-91.7)$ & $87.7(5.2)$ & $87.5(4.8)$ \\
\hline Sleep onset (hr:min) & $00: 30(78)$ & 00:30 (78) & 00:30 (78) & 00:00 (23:30-01:00) & $00: 30(78)$ & $00: 30(78)$ \\
\hline Wake (hr:min) & 07:55 (72) & 07:55 (72) & 08:00 (72) & 08:00 (90) & 07:55 (72) & 07:55 (1.2) \\
\hline
\end{tabular}

Note. Data are presented as mean or median (SD or 25 th-75th percentile).

Table 2 ICCs and Agreement Between Pairs of Monitors Within Brands and Between Wrists (ND AX/D AX, ND GEN/ D GEN, ND ActiG/D ActiG) for Sleep Time, Sleep Window, Sleep Efficiency, Sleep Onset, and Wake Time

\begin{tabular}{|c|c|c|c|c|c|c|c|c|c|}
\hline \multirow[b]{2}{*}{ Monitor } & \multicolumn{3}{|c|}{$\mathrm{ICC}^{\mathrm{a}}(95 \% \mathrm{Cl})$} & \multicolumn{3}{|c|}{$\begin{array}{l}\text { Agreement (Bland and } \\
\text { Altman), }{ }^{b} \text { mean bias }\end{array}$} & \multicolumn{3}{|c|}{$95 \%$ limits of agreement } \\
\hline & $\mathbf{A X}$ & GEN & ActiG & $\mathbf{A X}$ & GEN & ActiG & $\mathbf{A X}$ & GEN & ActiG \\
\hline Sleep time (min) & $.68[.50, .81]$ & $.84[.74, .91]$ & $.91[.86, .95]$ & -3.5 & -5.7 & -1.7 & 86.7 & 54.6 & 39.5 \\
\hline Sleep window (min) & $.56[.32, .73]$ & $.80[.66, .88]$ & $.85[.75, .91]$ & -1.3 & -3.1 & 2.3 & 102.9 & 66.1 & 54.2 \\
\hline Sleep efficiency $(\%)$ & $.82[.71, .90]$ & $.87[.77, .93]$ & $.88[.78, .93]$ & -0.4 & -0.6 & -0.8 & 5.6 & 4.5 & 4.0 \\
\hline Sleep onset (min) & $.88[.79, .93]$ & $.96[.94, .98]$ & $.97[.96, .99]$ & 3.0 & 3.6 & 0.0 & 90.0 & 48.6 & 40.2 \\
\hline Wake (min) & $.89[.81, .94]$ & $.96[.93, .98]$ & $.97[.95, .98]$ & 1.2 & 0.6 & 2.4 & 78.0 & 44.4 & 38.8 \\
\hline
\end{tabular}

Note. $\mathrm{ND}=$ nondominant; $\mathrm{D}=$ dominant; $\mathrm{AX}=$ Axivity; GEN = GENEActiv; ActiG = ActiGraph GT9X; ICC = intraclass correlation; CI = confidence interval.

${ }^{\mathrm{a}}$ Single measure, absolute agreement. All $p \mathrm{~s}<.05$. ${ }^{\mathrm{b}}$ Bland and Altman, bias calculated as nondominant - dominant. 

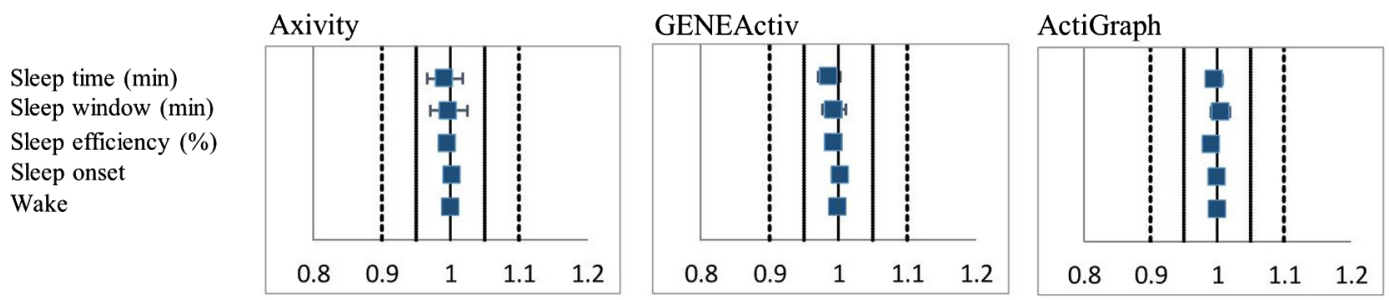

Figure 1 - Equivalence between pairs of monitors between wrists and within brand. Error bars represent the $95 \% \mathrm{CI}$ of the ratio. Equivalence $=1$ (solid line). Dashed lines indicate the $10 \%$ equivalence zone, and thick solid lines indicate a 5\% equivalence zone. Markers in black indicate if outcomes are within the $10 \%$ and $5 \%$ equivalence zones; light gray indicates if outcomes within the $10 \%$ equivalence zone but not the $5 \%$. CI $=$ confidence interval.

Table 3 ICCs and Agreement Between Pairs of Monitors Within Nondominant and Dominant Wrists Between Brands for Sleep Time, Sleep Window, Sleep Efficiency, Sleep Onset, and Wake Time

\begin{tabular}{|c|c|c|c|c|c|c|c|c|c|}
\hline \multirow[b]{2}{*}{ Monitor 1} & \multicolumn{3}{|c|}{$\mathrm{ICC}^{\mathrm{a}}(95 \% \mathrm{Cl})$} & \multicolumn{3}{|c|}{$\begin{array}{l}\text { Agreement (Bland and } \\
\text { Altman), }{ }^{b} \text { mean bias }\end{array}$} & \multicolumn{3}{|c|}{$95 \%$ limits of agreement } \\
\hline & $\mathbf{A X}$ & $\mathrm{AX}$ & GEN & AX & $A X$ & GEN & $\overline{A X}$ & $\mathbf{A X}$ & GEN \\
\hline Monitor 2 & GEN & ActiG & ActiG & GEN & ActiG & ActiG & GEN & ActiG & ActiG \\
\hline \multicolumn{10}{|l|}{ Nondominant wrist } \\
\hline Sleep time (min) & $.89[.81, .94]$ & $.91[.85, .95]$ & $.93[.89, .96]$ & -5.0 & -3.1 & -1.9 & 45.2 & 39.1 & 34.4 \\
\hline Sleep window (min) & $.85[.75, .91]$ & $.86[.76, .92]$ & $.93[.89, .96]$ & -7.4 & -5.3 & -2.1 & 56.2 & 53.1 & 37.5 \\
\hline Sleep efficiency $(\%)$ & $.87[.77, .92]$ & $.95[.91, .97]$ & $.88[.79, .92]$ & 0.2 & 0.3 & -0.1 & 4.4 & 2.5 & 4.3 \\
\hline Sleep onset (min) & $.97[.94, .98]$ & $.98[.97, .99]$ & $.97[.95, .98]$ & 1.2 & 4.4 & -3.0 & 46.8 & 32.2 & 41.8 \\
\hline Wake (min) & $.96[.92, .97]$ & $.96[.93, .98]$ & $.96[.92, .98]$ & -6.2 & -1.2 & -5.4 & 47.6 & 45.0 & 45.3 \\
\hline \multicolumn{10}{|l|}{ Dominant wrist } \\
\hline Sleep time (min) & $.76[.61, .86]$ & $.78[.63, .87]$ & $.91[.84, .95]$ & -7.2 & -1.3 & -5.9 & 75.2 & 72.8 & 40.2 \\
\hline Sleep window $(\mathrm{min})$ & $.69[.51, .81]$ & $.73[.56, .84]$ & $.85[.75, .92]$ & -9.2 & -1.7 & -7.5 & 83.4 & 78.1 & 51.8 \\
\hline Sleep efficiency (\%) & $.87[.78, .93]$ & $.93[.88, .96]$ & $.92[.86, .96]$ & 0.0 & -0.1 & 0.1 & 5.2 & 3.8 & 3.7 \\
\hline Sleep onset (min) & $.92[.86, .95]$ & $.91[.85, .95]$ & $.99[.98, .99]$ & 2.0 & 1.7 & 0.3 & 75.1 & 76.2 & 27.6 \\
\hline Wake (min) & $.90[.82, .94]$ & $.91[.84, .95]$ & $.96[.93, .98]$ & -7.2 & 0.0 & -7.2 & 74.2 & 71.1 & 42.6 \\
\hline
\end{tabular}

Note. AX = Axivity; GEN = GENEActiv; ActiG = ActiGraph GT9X; ICC = intraclass correlation; $\mathrm{CI}=$ confidence interval.

${ }^{\mathrm{a}}$ Single measure, absolute agreement. All $p s<.05$. ${ }^{\mathrm{b}}$ Bland and Altman, bias calculated as Monitor $1-$ Monitor 2.

The lowest mean bias for all sleep outcomes and narrowest $95 \%$ LoA for sleep time and sleep window were observed between the GENEActiv/ActiGraph pairing. The width of $95 \%$ LoA for sleep onset (up to $\pm 48.0 \mathrm{~min}$ ) and wake time (up to $\pm 48.0 \mathrm{~min}$ ) was similar across all pairings of the monitors. Sleep outcomes were within the proposed $5 \%$ and $10 \%$ equivalence (Figure $2 \mathrm{a}$ ).

Dominant placement. Reliability was good to excellent and higher between the GENEActiv/ActiGraph pairing when worn on the dominant wrist, with ICCs of .85-.99 compared to the Axivity/ActiGraph (ICCs of .73-.93) and Axivity/GENEActiv (.69-.92). Although low mean bias were observed between pairs of monitors for all sleep outcomes, wide 95\% LoA were observed for sleep time, sleep window, sleep onset, and wake time outcomes across three pairs of monitors, with the lowest between the GENEActiv/ActiGraph pairing. All sleep outcomes were within the $5 \%$ and $10 \%$ equivalence zones between all pairs of monitors (Figure 2b).

\section{Between Wrists and Between Brands}

The sleep outcome results between different brands between the nondominant and dominant wrists are presented in Table 4, and
Bland-Altman plots are presented in Supplementary Figure S3 (available online). The level of reliability varied from poor to good between all pairings of monitors for sleep time (ICCs of .66-.88) and sleep window (ICCs of .57-.80) and was moderate to excellent for sleep efficiency (ICCs of .76-.89), sleep onset (ICCs of .88.97 ), and sleep window (ICCs of .88-.97). Similar reliability was found between the dominant Axivity/nondominant GENEActiv and dominant Axivity/nondominant ActiGraph pairings and was lowest compared with other pairings of the monitors. The mean bias between pairs of monitors for all sleep outcomes was low; however, high 95\% LoA for sleep time (up to $\pm 90.1 \mathrm{~min}$ ), sleep window $( \pm 102.1 \mathrm{~min})$, sleep onset $( \pm 90.0 \mathrm{~min})$, and wake time $( \pm 84.0 \mathrm{~min})$ were observed. Sleep outcomes from any monitor combination fell within the proposed equivalence (see Figure 3).

\section{With and Without Using Sleep Log Data}

Results from the monitors when using and not using sleep log data are shown in Table 5 and the Bland-Altman plots in Supplementary Figure S4a-S4c (available online). Overall, the level of agreement appeared slightly higher at the dominant location compared with the nondominant location for all sleep 
(a)

Sleep time ( $\mathrm{min})$ Sleep window $(\mathrm{min})$ Sleep efficiency $(\%)$ Sleep onset

Wake a)

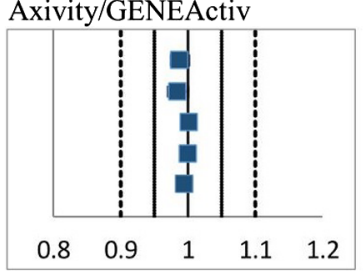

(b)

Sleep time $(\mathrm{min})$ Sleep window (min) Sleep efficiency $(\%)$ Sleep onset Wake

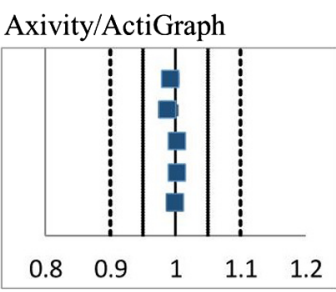

GENEActiv/ActiGraph

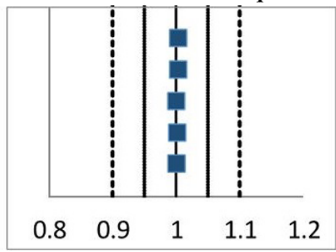

GENEActiv/ActiGraph

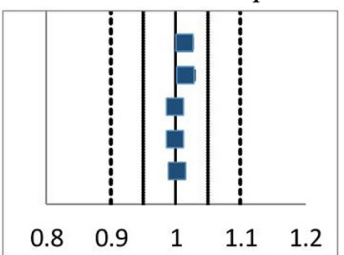

Figure 2 - Equivalence between pairs of monitors within the wrists and between brands worn on the (a) nondominant wrist and (b) dominant wrist. Error bars represent the $95 \% \mathrm{CI}$ of the ratio. Equivalence $=1$ (solid line). Dashed lines indicate the $10 \%$ equivalence zone, and thick solid lines indicate the $5 \%$ equivalence zone. Markers in black indicate if outcomes are within the $10 \%$ and $5 \%$ equivalence zones; light gray indicates if outcomes are within the $10 \%$ equivalence zone but not the $5 \%$. $\mathrm{CI}=$ confidence interval.

Table 4 ICCs and Agreement Between Pairs of Monitors Between Wrists Between Brands for Sleep Time, Sleep Window, Sleep Efficiency, Sleep Onset, and Wake Time

\begin{tabular}{|c|c|c|c|c|c|c|c|c|c|}
\hline \multirow[b]{2}{*}{ Monitor 1} & \multicolumn{3}{|c|}{$I C^{a}(95 \%$ CI) } & \multicolumn{3}{|c|}{$\begin{array}{l}\text { Agreement (Bland and } \\
\text { Altman), }{ }^{\mathbf{b}} \text { mean bias }\end{array}$} & \multicolumn{3}{|c|}{$95 \%$ limits of agreement } \\
\hline & ND AX & ND AX & ND GEN & ND AX & ND AX & ND GEN & ND $A X$ & ND $A X$ & ND GEN \\
\hline Monitor 2 & D GEN & D ActiG & D ActiG & D GEN & D ActiG & D ActiG & D GEN & D ActiG & D ActiG \\
\hline Sleep time (min) & $.86[.74, .93]$ & $.86[.76, .92]$ & $.82[.69, .89]$ & -10.7 & -4.8 & 0.2 & 47.6 & 50.7 & 59.0 \\
\hline Sleep window (min) & $.79[.64, .88]$ & $.80[.66, .88]$ & $.76[.61, .86]$ & -10.5 & 3.0 & 4.4 & 63.7 & 64.3 & 70.4 \\
\hline Sleep efficiency $(\%)$ & $.86[.77, .92]$ & $.89[.81, .94]$ & $.80[.67, .88]$ & -0.4 & -0.5 & -0.6 & 4.5 & 3.9 & 5.5 \\
\hline Sleep onset (min) & $.97[.94, .98]$ & $.97[.95, .98]$ & $.96[.92, .98]$ & 4.8 & 4.5 & 3.3 & 44.7 & 43.0 & 52.4 \\
\hline Wake (min) & $.97[.94, .98]$ & $.97[.94, .98]$ & $.95[.91, .97]$ & -5.8 & 1.5 & 7.7 & 40.6 & 41.7 & 48.0 \\
\hline Monitor 1 & D AX & D AX & D GEN & $D A X$ & D AX & D GEN & D AX & $\mathbf{D A X}$ & D GEN \\
\hline Monitor 2 & ND GEN & ND ActiG & ND ActiG & $\overline{\text { ND GEN }}$ & $\overline{\text { ND ActiG }}$ & $\overline{\text { ND ActiG }}$ & $\overline{\text { ND GEN }}$ & $\overline{\text { ND ActiG }}$ & $\overline{\text { ND ActiG }}$ \\
\hline Sleep time (min) & $.66[.46, .80]$ & $.70[.52, .82]$ & $.88[.78, .93]$ & -1.5 & 0.4 & 5.9 & 90.1 & 83.4 & 40.2 \\
\hline Sleep window (min) & $.57[.34, .74]$ & $.59[.37, .75]$ & $.79[.66, .88]$ & -6.1 & -4.0 & 5.2 & 102.1 & 96.8 & 63.7 \\
\hline Sleep efficiency $(\%)$ & $.76[.61, .86]$ & $.81[.69, .89]$ & $.83[.70, .90]$ & 0.6 & 0.7 & 0.7 & 6.8 & 5.7 & 5.1 \\
\hline Sleep onset (min) & $.88[.79, .93]$ & $.89[.81, .94]$ & $.97[.95, .98]$ & -1.5 & 1.2 & -0.4 & 91.2 & 87.3 & 43.1 \\
\hline Wake (min) & $.88[.82, .94]$ & $.88[.84, .95]$ & $.96[.93, .98]$ & -7.8 & -2.4 & 4.8 & 81.8 & 81.8 & 46.5 \\
\hline
\end{tabular}

Note. $\mathrm{ND}=$ nondominant; $\mathrm{D}=$ dominant; $\mathrm{AX}=$ Axivity; GEN = GENEActiv; ActiG = ActiGraph GT9X; ICC = intraclass correlation; CI = confidence interval.

${ }^{\mathrm{a}}$ Single measure, absolute agreement. All $p_{s}<.05$. ${ }^{\mathrm{b}}$ Bland and Altman, bias calculated as Monitor $1-$ Monitor 2.

outcomes. Reliability was poor for sleep time (ICCs of .26-.91) and sleep window (ICCs of .24-.86) for all accelerometer brands regardless of the placement of the monitor, with an exception of dominant placement of the Axivity showing moderate reliability. Sleep time, sleep window, and wake time appeared higher and sleep onset lower in the no sleep log data condition compared with the sleep log data condition irrespective of wrist in all accelerometer brands. All sleep outcomes were within the $10 \%$ equivalence zone from each brand of monitor irrespective of sleep log condition (Figure $4 \mathrm{a}-4 \mathrm{c}$ ). However, sleep time and sleep window outcomes were within the $5 \%$ equivalence zone across all monitor pairings.

\section{Discussion}

This study aimed to establish whether sleep outcomes were comparable between three commonly used accelerometer brands when worn on the dominant and nondominant wrists and with or without the use of self-reported sleep log data. This was achieved by identically analyzing data with a freely available GGIR package, both with a sleep log and using the HDCZA (van Hees et al., 2018).

Sleep outcomes were within the $10 \%$ equivalency zone between any combination of the monitors and irrespective of the availability of sleep log data. Reliability between the pairs 

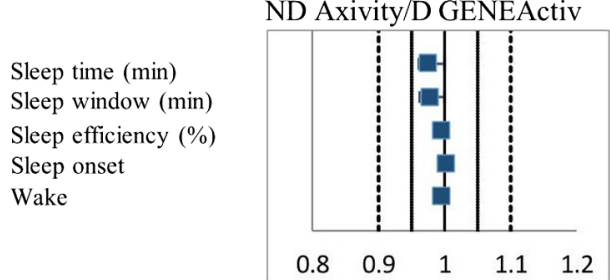

D Axivity/ND GENEActiv

Sleep time $(\mathrm{min})$

Sleep window $(\mathrm{min})$

Sleep efficiency $(\%)$

Sleep onset

Wake

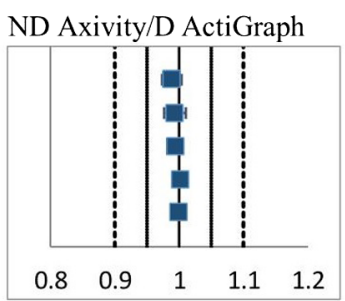

D Axivity/ND ActiGraph

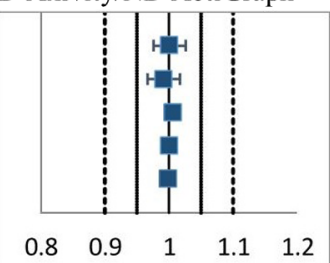

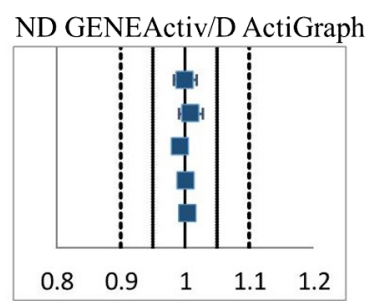

D GENEActiv/ND ActiGraph

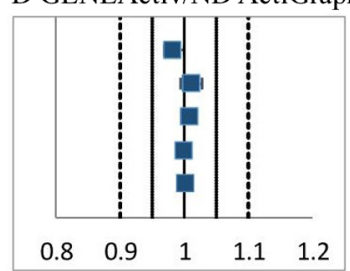

Figure 3 - Equivalence between pairs of monitors between wrists and between brands. Error bars represent the $95 \% \mathrm{CI}$ of the ratio. Equivalence $=1$ (solid line). Dashed lines indicate the $10 \%$ equivalence zone, and thick solid lines indicate the 5\% equivalence zone. Markers in black indicate if outcomes are within the $10 \%$ and $5 \%$ equivalence zones; light gray indicates if outcomes are within the $10 \%$ equivalence zone but not the $5 \%$. CI $=$ confidence interval; $\mathrm{ND}=$ nondominant; $\mathrm{D}=$ dominant.

Table 5 ICCs and Agreement Between Nondominant and Dominant Wrists Within Axivity, GENEActiv, and ActiGraph With and Without Sleep Log for Sleep Time, Sleep Window, Sleep Efficiency, Sleep Onset, and Wake Time

\begin{tabular}{|c|c|c|c|c|c|c|}
\hline & \multirow{2}{*}{\multicolumn{2}{|c|}{$\mathrm{ICC}^{\mathrm{a}}(95 \% \mathrm{Cl})$}} & \multicolumn{4}{|c|}{ Agreement (Bland and Altman) $^{\mathrm{b}}$} \\
\hline & & & \multicolumn{2}{|c|}{ Nondominant } & \multicolumn{2}{|c|}{ Dominant } \\
\hline & Nondominant & Dominant & Mean bias & $95 \%$ LoA & Mean bias & 95\% LoA \\
\hline \multicolumn{7}{|l|}{$\begin{array}{l}\text { Axivity sleep log versus } \\
\text { no sleep log }\end{array}$} \\
\hline Sleep time (min) & $.78[.46, .90]$ & $.83[.68, .90]$ & 20.0 & 57.2 & 14.3 & 66.9 \\
\hline Sleep window (min) & $.68[.34, .83]$ & $.70[.50, .82]$ & 22.5 & 77.8 & 16.0 & 85.6 \\
\hline Sleep efficiency $(\%)$ & $.84[.73, .91]$ & $.96[.92, .98]$ & 0.0 & 4.8 & 0.1 & 3.3 \\
\hline Sleep onset (min) & $.93[.86, .96]$ & $.90[.83, .95]$ & -7.2 & 67.5 & -11.1 & 72.0 \\
\hline Wake (min) & $.89[.78, .94]$ & $.93[.87, .96]$ & 20.4 & 68.5 & 17.7 & 67.7 \\
\hline \multicolumn{7}{|c|}{$\begin{array}{l}\text { GENEActiv sleep log versus } \\
\text { no sleep log }\end{array}$} \\
\hline Sleep time (min) & $.74[.35, .88]$ & $.77[.29, .91]$ & 23.0 & 59.0 & 24.8 & 55.0 \\
\hline Sleep window (min) & $.64[.28, .81]$ & $.69[.24, .86]$ & 27.6 & 81.5 & 28.8 & 69.9 \\
\hline Sleep efficiency $(\%)$ & $.86[.77, .92]$ & $.95[.91, .97]$ & -0.4 & 4.9 & -0.2 & 3.1 \\
\hline Sleep onset (min) & $.92[.86, .95]$ & $.93[.87, .96]$ & -6.8 & 59.7 & -8.4 & 74.6 \\
\hline Wake (min) & $.87[.69, .94]$ & $.88[.74, .94]$ & 15.7 & 69.3 & 7.6 & 65.8 \\
\hline \multicolumn{7}{|c|}{$\begin{array}{l}\text { ActiGraph sleep log versus } \\
\text { no sleep log }\end{array}$} \\
\hline Sleep time $(\min )$ & $.72[.26, .88]$ & $.78[.39, .90]$ & 24.9 & 58.3 & 21.7 & 55.0 \\
\hline Sleep window $(\mathrm{min})$ & $.61[.25, .80]$ & $.70[.32, .86]$ & 27.7 & 80.8 & 24.5 & 66.1 \\
\hline Sleep efficiency $(\%)$ & $.73[.56, .84]$ & $.91[.84, .95]$ & 0.1 & 6.6 & 0.1 & 4.0 \\
\hline Sleep onset (min) & $.93[.86, .96]$ & $.95[.90, .97]$ & -11.6 & 60.8 & -10.1 & 49.8 \\
\hline Wake (min) & $.87[.74, .93]$ & $.89[.79, .94]$ & 16.2 & 72.8 & 14.3 & 64.0 \\
\hline
\end{tabular}

Note. $\mathrm{LoA}=$ limits of agreement; $\mathrm{ICC}=$ intraclass correlation; $\mathrm{CI}=$ confidence interval.

${ }^{\mathrm{a}}$ Single measure, absolute agreement. All $p_{s}<.05$. ${ }^{\mathrm{b}}$ Bland and Altman, bias calculated as no sleep log condition - sleep log condition.

of accelerometers was mostly good to excellent. There was low level of mean bias; however, there were wide $95 \%$ LoA for sleep time, sleep window, sleep onset, and wake time outcomes, indicating that individual-level data should be compared with caution.
In addition to poor reliability, when applying a stricter 5\% equivalence zone, sleep time, and sleep window outcomes were not equivalent between the sleep $\log /$ no sleep log condition in all three accelerometer brands. The SPT window is derived from sleep onset 
(a) ND Axivity

Sleep time $(\mathrm{min})$ Sleep window $(\mathrm{min})$ Sleep efficiency $(\%)$ Sleep onset Wake

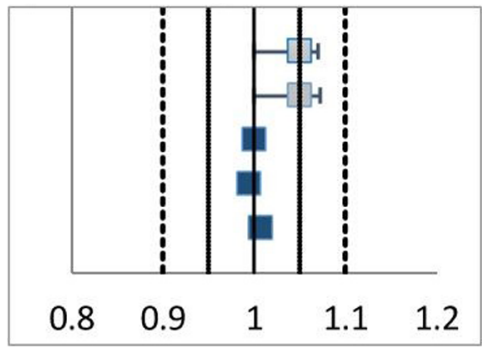

(b)

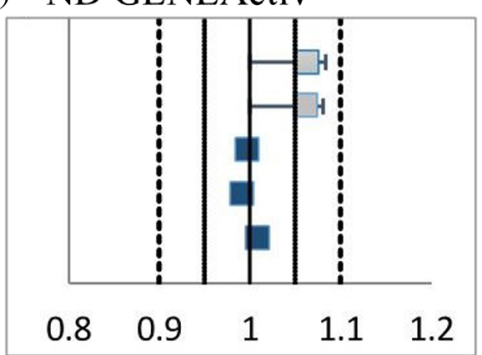

(c) ND ActiGraph

Sleep time $(\mathrm{min})$

Sleep window $(\mathrm{min})$

Sleep efficiency (\%)

Sleep onset

Wake

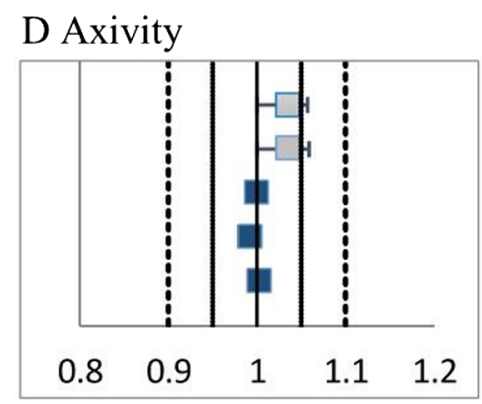

D GENEActiv

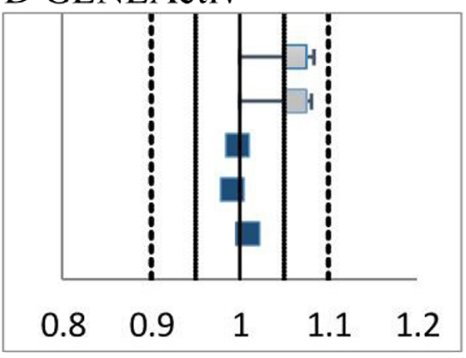

D ActiGraph

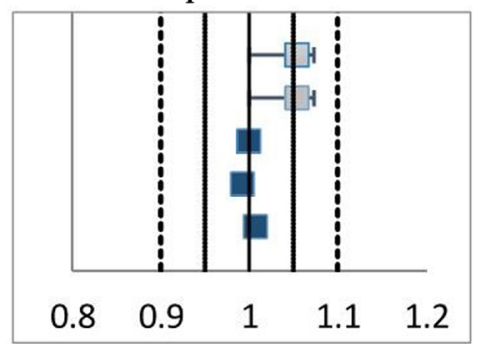

Figure $4-(\mathrm{a}-\mathrm{c})$ Equivalence between pairs of monitors within the wrists and within brands when using/not using a sleep log. Error bars represent the $95 \% \mathrm{CI}$ of the ratio. Equivalence $=1$ (solid line). Dashed lines indicate the $10 \%$ equivalence zone, and thick solid lines indicate the 5\% equivalence zone. Markers in black indicate if outcomes are within the $10 \%$ and $5 \%$ equivalence zones; light gray indicates if outcomes are within the $10 \%$ equivalence zone but not the $5 \%$. $\mathrm{CI}=$ confidence interval; $\mathrm{ND}=$ nondominant; $\mathrm{D}=$ dominant.

and waking time, and therefore lower agreement and reliability for sleep time and sleep window compared with other sleep outcomes could be due to misclassification of sleep duration within the SPT window. The better agreement observed for sleep efficiency compared with SPT window and sleep time is because the ratio between total sleep time/total minutes in bed ([sleep efficiency calculation sleep time/SPT window] $\times 100$ ) remained very similar. This is because both total sleep time and SPT window differed similarly between brands, resulting in similar ratios (sleep efficiency) between brands. Sleep efficiency is always calculated from the SPT window and sleep time variables that come from the same monitor.

The placement of the accelerometer has been proposed to affect physical activity outcomes as the dominant arm tends to be stronger and used more often than the nondominant for regular lifestyle activities (Dieu et al., 2017); subsequently, the nondominant wrist has been commonly used as the site of monitor placement, with a notable exception in the UK Biobank data set where the Axivity accelerometer was worn on the dominant wrist (Doherty et al., 2017). In this sample, average acceleration measured at the dominant wrist was higher compared with the nondominant wrist (Rowlands et al., 2019). Additionally, in this sample, the ActiGraph and GENEActiv demonstrated good to excellent ICCs for most physical activity outcomes (such as average acceleration and total moderate to vigorous physical activity [moderate to vigorous physical activity and moderate to vigorous physical activity in bouts]) between wrists (Rowlands et al., 2019). Similarly, ICCs and agreement for all sleep outcomes were higher for the ActiGraph, followed by the GENEActiv and Axivity, which had moderate to poor ICCs and noticeably wider LoA for sleep time, sleep window, sleep onset, and wake time outcomes compared with the other monitors. Studies that have validated the same brand of accelerometers (ActiGraph; Full et al., 2018) and actigraphy (Driller, O’Donnell, \& Tavares, 2017) against PSG showed no differences in sleep estimates between the nondominant and dominant wrists. The findings from the current study reassure that sleep data from the same accelerometer brand worn on either wrist collected in a free-living environment are comparable.

Overall, ICCs were good to excellent for all sleep outcomes between brands on either wrist, and irrespective of wrist, higher ICCs and agreement were observed between the ActiGraph/ GENEActiv compared with other pairings of accelerometers. 
Slightly better reliability and agreement appeared for all sleep outcomes when worn on nondominant wrist compared with dominant placement. Evidence from this sample showed that physical activity outcomes are mostly equivalent between the Axivity, GENEActiv, and ActiGraph accelerometers when worn on the nondominant wrist (Rowlands et al., 2019). When worn on the dominant wrist, equivalency for physical activity outcomes was poorer compared with nondominant placement; however, high reliability and agreement for physical activity outcomes were observed and appeared more consistent between the Axivity and GENEActiv (Rowlands et al., 2018, 2019). These discrepancies in equivalency have been proposed to be attributed to the technical differences between brands, which may also explain the differences in reliability and agreement for sleep outcomes (Rowlands et al., 2016).

Furthermore, the comparability of sleep outcomes was assessed in the absence and presence of sleep log data. A sleep log requires participants to record sleep and wake times and is used to guide sleep detection algorithms to identify the sleep period (van Hees et al., 2015). In this study, in addition to fairly low mean bias, the ICCs were mostly good for sleep onset and wake time, supporting the consistency of the algorithm to detect the SPT window without the aid of sleep log data across accelerometer brands (van Hees et al., 2018). However, regardless of accelerometer placement, sleep time and sleep window outcomes within the SPT window detected with and without a sleep log had mostly poor reliability and were not in the stricter $5 \%$ equivalence zone (which, of a typical night of 480 min, would represent $24 \mathrm{~min}$ ). Given the present findings, it is important to consider when comparing or pooling sleep data from different studies that differ on sleep log availability that sleep time and sleep window are less comparable than other sleep outcomes between sleep log and no sleep log condition.

This study has a number of strengths, including 24-hr activity assessment in free-living individuals for several days and simultaneous comparison of three different brands of accelerometers worn on the nondominant and dominant wrists. Importantly, data were analyzed using an open-source GGIR package in $\mathrm{R}$, which allows identical data handling and facilitates comparability of the results and can be easily applied to raw accelerometry data sets. This study has a number of limitations. First, this study only demonstrates comparability between sleep estimates between three commonly used accelerometers; it did not compare sleep estimates with PSG, the gold standard measure of sleep parameters. Second, the algorithm used in the current study is relatively new and therefore has undergone limited validation against PSG to date. Additionally, taping Axivity and ActiGraph together may have affected the results, although the agreement did not appear to be consistently higher between the Axivity/ActiGraph compared with the GENEActiv/ActiGraph. Future studies should consider different setups of accelerometers to establish the impact on agreement between monitors. Another limitation is the homogenous sample consisting of young healthy adults. The sleep algorithm used in this study was previously demonstrated to detect the SPT window better in healthy, good sleepers compared with those with sleep disorders (van Hees et al., 2018). Future studies should consider including different age groups and populations. Individual differences observed in this study could be addressed by collecting data on sleep symptomology and assessing the data over weekdays and weekends.

In conclusion, sleep outcomes from Axivity, GENEActiv, and ActiGraph accelerometers generated using the GGIR package were comparable when worn on either wrist irrespective of the accelerometer brand when the sleep log was not used. Findings from the present study support the comparison of sleep data from different accelerometers at the population level; however, caution is required if interest is in individual-level sleep data and if studies differ on the availability of a sleep $\log$.

\section{Acknowledgments}

The authors would like to acknowledge Elysa Ioannou, Francesca Denton, and Shinelle Baptiste for assisting with data collection and thank all participants who volunteered to take part in this study. The research was supported by the National Institute for Health Research (NIHR) Biomedical Research Centre based at University Hospitals of Leicester and Loughborough University, the National Institute for Health Research Collaboration for Leadership in Applied Health Research and Care-East Midlands (NIHR CLAHRC-EM). The views expressed are those of the authors and not necessarily those of the NHS, the NIHR, or the Department of Health. No external sources of funding were accessed. Authors declared no conflict of interest. Anonymized data sets generated during this study are available on reasonable request. Requests for access to data can be sent to the corresponding author.

\section{References}

Berger, A.M., Wielgus, K.K., Young-McCaughan, S., Fischer, P., Farr, L., \& Lee, K.A. (2008). Methodological challenges when using actigraphy in research. Journal of Pain and Symptom Management, 36(2), 191199. PubMed ID: 18400460 doi:10.1016/j.jpainsymman.2007.10.008

Bland, J.M., \& Altman, D. (1986). Statistical methods for assessing agreement between two methods of clinical measurement. The Lancet, 327(8476), 307-310. doi:10.1016/S0140-6736(86)90837-8

Boddy, L.M., Noonan, R.J., Kim, Y., Rowlands, A.V., Welk, G.J., Knowles, Z.R., \& Fairclough, S.J. (2018). Comparability of children's sedentary time estimates derived from wrist worn GENEActiv and hip worn ActiGraph accelerometer thresholds. Journal of Science and Medicine in Sport, 21(10), 1045-1049. PubMed ID: 29650338 doi:10.1016/j.jsams.2018.03.015

Borazio, M., Berlin, E., Kücükyildiz, N., Scholl, P., \& Van Laerhoven, K. (2014, September). Towards benchmarked sleep detection with wrist-worn sensing units. Paper presented at the 2014 IEEE International Conference on Healthcare Informatics (pp. 125-134), Verona, Italy.

Cole, R.J., Kripke, D.F., Gruen, W., Mullaney, D.J., \& Gillin, J.C. (1992). Automatic sleep/wake identification from wrist activity. Sleep, 15(5), 461-469. PubMed ID: 1455130 doi:10.1093/sleep/15.5.461

da Silva, I.C., van Hees, V.T., Ramires, V.V., Knuth, A.G., Bielemann, R.M., Ekelund, U., . . Hallal, P.C. (2014). Physical activity levels in three Brazilian birth cohorts as assessed with raw triaxial wrist accelerometry. International Journal of Epidemiology, 43(6), 1959-1968. PubMed ID: 25361583 doi:10.1093/ije/dyu203

Dieu, O., Mikulovic, J., Fardy, P.S., Bui-Xuan, G., Béghin, L., \& Vanhelst, J. (2017). Physical activity using wrist-worn accelerometers: Comparison of dominant and non-dominant wrist. Clinical Physiology and Functional Imaging, 37(5), 525-529. PubMed ID: 26749436 doi: 10.1111/cpf.12337

Doherty, A., Jackson, D., Hammerla, N., Plötz, T., Olivier, P., Granat, M.H., ... Owen, C.G. (2017). Large scale population assessment of physical activity using wrist worn accelerometers: The UK Biobank Study. PLoS One, 12(2), e0169649. PubMed ID: 28146576 doi:10. 1371/journal.pone.0169649

Driller, M.W., O’Donnell, S., \& Tavares, F. (2017). What wrist should you wear your actigraphy device on? Analysis of dominant vs. non-dominant wrist actigraphy for measuring sleep in healthy adults. Sleep 
Science, 10(3), 132-135. PubMed ID: 29410743 doi:10.5935/19840063.20170023

Full, K.M., Kerr, J., Grandner, M.A., Malhotra, A., Moran, K., Godoble, S., ... Soler, X. (2018). Validation of a physical activity accelerometer device worn on the hip and wrist against polysomnography. Sleep Health, 4(2), 209-216. PubMed ID: 29555136 doi:10.1016/j.sleh. 2017.12.007

Girschik, J., Fritschi, L., Heyworth, J., \& Waters, F. (2012). Validation of self-reported sleep against actigraphy. Journal of Epidemiology, 22(5), 462-468. PubMed ID: 22850546 doi:10.2188/jea.JE20120012

Knutson, K.L., \& von Schantz, M. (2018). Associations between chronotype, morbidity and mortality in the UK Biobank cohort. Chronobiology International, 35(8), 1-9. doi:10.1080/07420528.2018.1454458

Koo, T.K., \& Li, M.Y. (2016). A guideline of selecting and reporting intraclass correlation coefficients for reliability research. Journal of Chiropractic Medicine, 15(2), 155-163. PubMed ID: 27330520 doi:10.1016/j.jcm.2016.02.012

Lauderdale, D.S., Knutson, K.L., Yan, L.L., Liu, K., \& Rathouz, P.J. (2008). Sleep duration: How well do self-reports reflect objective measures? The CARDIA Sleep Study. Epidemiology, 19(6), 838845. doi:10.1097/EDE.0b013e318187a7b0

Lee, J., Kim, Y., \& Welk, G.J. (2014). Validity and utility of consumerbased physical activity monitors. Medicine \& Science in Sports \& Exercise, 46(9), 1840-1848. PubMed ID: 24777201 doi:10.1249/ MSS.0000000000000287

Loprinzi, P.D., \& Cardinal, B.J. (2011). Association between objectivelymeasured physical activity and sleep, NHANES 2005-2006. Mental Health and Physical Activity, 4(2), 65-69. doi:10.1016/j.mhpa.2011. 08.001

Martin, J.L., \& Hakim, A.D. (2011). Wrist actigraphy. Chest, 139(6), 1514-1527. PubMed ID: 21652563 doi:10.1378/chest.10-1872

Menai, M., Van Hees, V.T., Elbaz, A., Kivimaki, M., Singh-Manoux, A., \& Sabia, S. (2017). Accelerometer assessed moderate-to-vigorous physical activity and successful ageing: Results from the Whitehall II study. Scientific Reports, 7(1), 45772. doi:10.1038/srep45772

Montoye, A.H., Nelson, M.B., Bock, J.M., Imboden, M.T., Kaminsky, L.A., Mackintosh, K.A., . . Pfeiffer, K.A. (2018). Raw and count data comparability of hip-worn ActiGraph GT3X and link accelerometers. Medicine \& Science in Sports \& Exercise, 50(5), 11031112. PubMed ID: 29283934 doi:10.1249/MSS.0000000000001534

Quante, M., Kaplan, E.R., Cailler, M., Rueschman, M., Wang, R., Weng, J., ... Redline, S. (2018). Actigraphy-based sleep estimation in adolescents and adults: A comparison with polysomnography using two scoring algorithms. Nature and Science of Sleep, 10, 13-20. PubMed ID: 29403321 doi:10.2147/NSS.S151085

Reutrakul, S., \& Van Cauter, E. (2018). Sleep influences on obesity, insulin resistance, and risk of type 2 diabetes. Metabolism, 84, 56-66. PubMed ID: 29510179 doi:10.1016/j.metabol.2018.02.010
Rosenberger, M.E., Buman, M.P., Haskell, W.L., McConnell, M.V., \& Carstensen, L.L. (2016). 24 hours of sleep, sedentary behavior, and physical activity with nine wearable devices. Medicine \& Science in Sports \& Exercise, 48(3), 457-465. PubMed ID: 26484953 doi:10. 1249/MSS.0000000000000778

Rowlands, A.V., Mirkes, E.M., Yates, T., Clemes, S., Davies, M., Khunti, K., \& Edwardson, C.L. (2018). Accelerometer-assessed physical activity in epidemiology: Are monitors equivalent? Medicine \& Science in Sports \& Exercise, 50(2), 257-265. PubMed ID: 28976493 doi:10.1249/MSS.0000000000001435

Rowlands, A.V., Plekhanova, T., Yates, T., Mirkes, E., Davies, M., Khunti, K., \& Edwardson, C. (2019). A basis for harmonisation of accelerometer physical activity outcomes in epidemiology. Journal for the Measurement of Physical Behaviour, 2(3), 131-142. doi:10. 1123/jmpb.2018-0073

Rowlands, A.V., Yates, T., Davies, M., Khunti, K., \& Edwardson, C.L. (2016). Raw accelerometer data analysis with GGIR R-package: Does accelerometer brand matter? Medicine \& Science in Sports \& Exercise, 48(10), 1935-1941. PubMed ID: 27183118 doi:10.1249/MSS. 0000000000000978

Sadeh, A. (2011). The role and validity of actigraphy in sleep medicine: An update. Sleep Medicine Reviews, 15(4), 259-267. PubMed ID: 21237680 doi:10.1016/j.smrv.2010.10.001

van Hees, V.T., Fang, Z., Langford, J., Assah, F., Mohammad, A., da Silva, I.C., ... Brage, S. (2014). Auto-calibration of accelerometer data for free-living physical activity assessment using local gravity and temperature: An evaluation on four continents. American Journal of Physiology-Heart and Circulatory Physiology, 117(7), 738-744.

van Hees, V.T., Gorzelniak, L., Dean León, E.C., Eder, M., Pias, M., Taherian, S., ... Brage, S. (2013). Separating movement and gravity components in an acceleration signal and implications for the assessment of human daily physical activity. PLoS One, 8(4), e61691. doi:10.1371/journal.pone.0061691

van Hees, V.T., Sabia, S., Anderson, K.N., Denton, S.J., Oliver, J., Catt, M., ... Singh-Manoux, A. (2015). A novel, open access method to assess sleep duration using a wrist-worn accelerometer. PLoS One, 10(11), e0142533. PubMed ID: 26569414 doi:10.1371/journal.pone. 0142533

van Hees, V.T., Sabia, S., Jones, S.E., Wood, A.R., Anderson, K.N., Kivimaki, M., ... Mazzotti, D.R. (2018). Estimating sleep parameters using an accelerometer without sleep diary. Scientific Reports, 8(1), 12975. PubMed ID: 30154500 doi:10.1038/s41598-018-31266-Z

Zinkhan, M., Berger, K., Hense, S., Nagel, M., Obst, A., Koch, B., ... Young, P. (2014). Agreement of different methods for assessing sleep characteristics: A comparison of two actigraphs, wrist and hip placement, and self-report with polysomnography. Sleep Medicine, 15(9), 1107-1114. PubMed ID: 25018025 doi:10.1016/j.sleep.2014. 04.015 